This item was submitted to Loughborough's Research Repository by the author.

Items in Figshare are protected by copyright, with all rights reserved, unless otherwise indicated.

\title{
Simultaneous measurement of in-plane and out-of-plane displacement fields in scattering media using phase-contrast spectral optical coherence tomography
}

\section{PLEASE CITE THE PUBLISHED VERSION}

\section{PUBLISHER}

(C) Optical Society of America

\section{VERSION}

AM (Accepted Manuscript)

LICENCE

CC BY-NC-ND 4.0

\section{REPOSITORY RECORD}

De la Torre Ibarra, Manuel H., Pablo D. Ruiz, and Jonathan M. Huntley. 2019. "Simultaneous Measurement of In-plane and Out-of-plane Displacement Fields in Scattering Media Using Phase-contrast Spectral Optical Coherence Tomography". figshare. https://hdl.handle.net/2134/5929. 
This item was submitted to Loughborough's Institutional Repository (https://dspace.lboro.ac.uk/) by the author and is made available under the following Creative Commons Licence conditions.

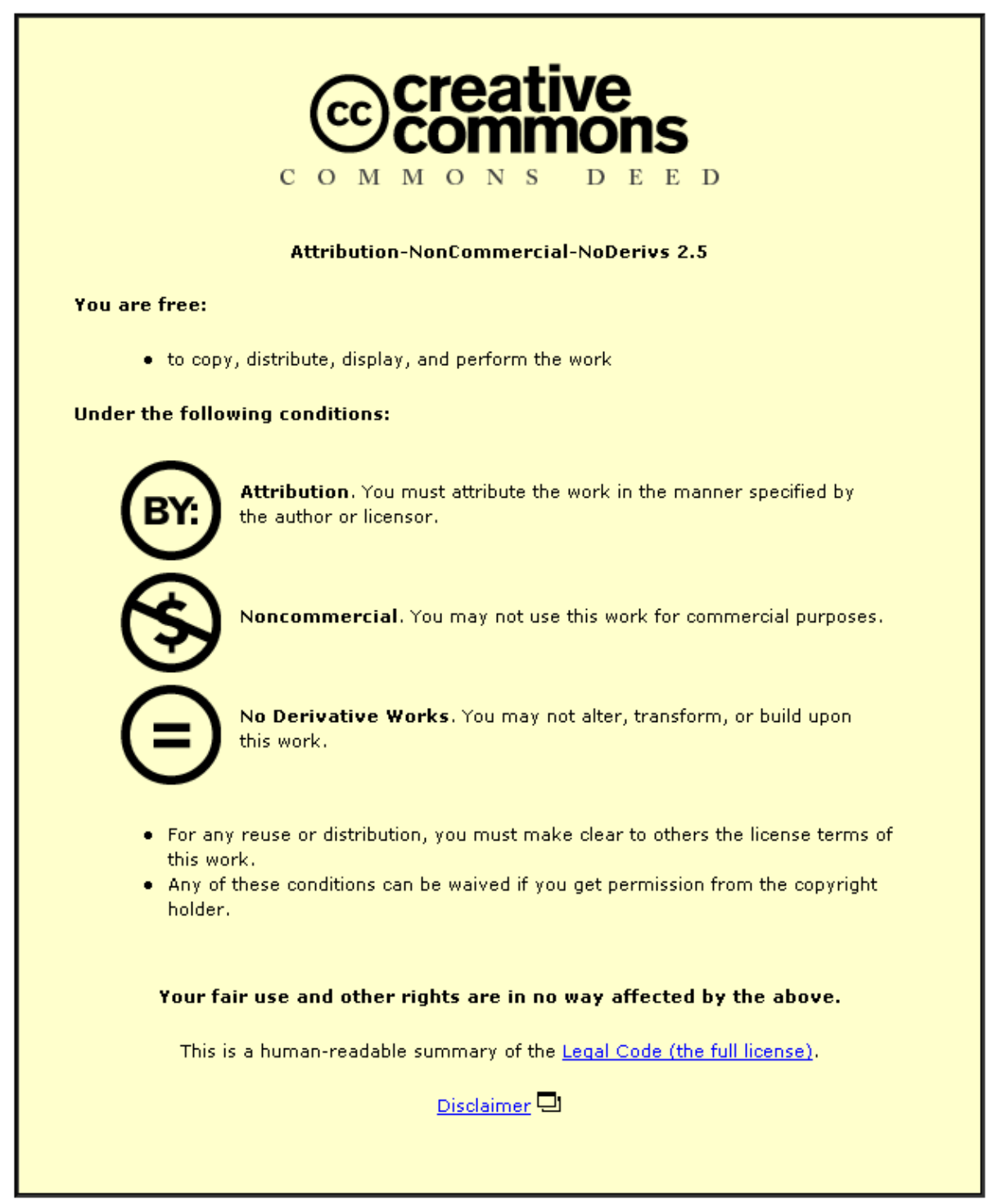

For the full text of this licence, please go to: http://creativecommons.org/licenses/by-nc-nd/2.5/ 


\title{
Simultaneous measurement of in-plane and out-of-
}

\section{plane displacement fields in scattering media using}

\section{phase contrast spectral optical coherence tomography}

\author{
Manuel H. De la Torre Ibarra ${ }^{1}$, Pablo D. Ruiz ${ }^{2}$, Jonathan M. Huntley² \\ ${ }^{1}$ Centro de Investigaciones en Óptica Asociación Civil, Loma Del Bosque 115, León Guanajuato C.P. \\ 37150, México \\ mandlti@cio.mx \\ ${ }^{2}$ Wolfson School of Mechanical and Manufacturing Engineering, Loughborough University, Ashby \\ Road, Loughborough, Leicestershire LE11 3TU, UK
}

\begin{abstract}
The use of phase contrast spectral optical coherence tomography to measure two orthogonal displacement components on a slice within a scattering medium is demonstrated. This is achieved by combining sequential oblique illumination of the object and recording two interferograms before plus two after the deformation. The proposed technique is illustrated with results from a sample undergoing simple shear. Depth-resolved out-of-plane and in-plane sensitivities of 0.14 and $4.2 \mu \mathrm{m}$ per fringe are demonstrated up to a depth of $400 \mu \mathrm{m}$ in a water based polymer.
\end{abstract}

OCIS codes:

(110.4500) Optical coherence tomography, (120.5050) Phase measurement, (120.3940) Metrology 
A diverse range of techniques, including for example electronic speckle pattern interferometry and digital holography, provide full-field non-contact displacement maps of the surface response of components to external loads [1]. A major limitation, however, is that only indirect information can be obtained about the component's internal deformation state. A second family of interferometric techniques known as optical coherence tomography (OCT) has been developed since the early 1990s for imaging within weakly scattering media. Traditional OCT applications are mostly medical and the phase data has tended to be neglected in comparison with the magnitude data which encodes the sample microstructure [2]. The idea of combining the strengths of both families (i.e., the high displacement sensitivity of speckle interferometry with the depth sensing capabilities of OCT) has been recently investigated by a number of authors in the field of optical metrology [3-7]. One of the most promising of these techniques is phase contrast spectral OCT which, without any moving parts, has the ability to generate a 2D tomographic image. Depth-resolved phase information encoded in the recorded data enables the evaluation of the displacement field in the depth-resolved slice. Conventional spectral OCT setups are based on coaxial illumination and observation of the sample, which only provides outof-plane sensitivity [7]. In this letter we present an optical system which can measure both in-plane and out-of-plane depth-resolved displacements with interferometric sensitivity within a scattering object in the same acquisition process.

Figure 1(a) shows a schematic view of the optical setup. The output of a super luminescent diode (SLD) (Superlum S840-HP-I, 15mW, central wavelength $\lambda_{\mathrm{c}}=$ 840nm and bandwidth $\Delta \lambda=50 \mathrm{~nm}$ ) is combined with a He-Ne laser used for 
alignment purposes $\left(30 \mathrm{~mW}, \lambda_{\mathrm{H}}=632.8 \mathrm{~nm}\right.$ ) with a 10:90 $2 \times 2$ optical fibre coupler. Reference and object beams are combined with a 10:90 non-polarizing beam splitter (BS), diffracted by a blazed grating $\mathrm{G}$ (grating frequency $=1200$ lines $\mathrm{mm}^{-1}$ ) and detected with a 2D CCD array (Vosskühler 1300QLN, 1280×1024 pixels, 12 bit resolution). An unfolded side view of the illumination beam is shown in more detail in Figure 1(b). This setup allows one on the one hand to illuminate the object with a light 'wedge' (focused on plane $x y$ ), and on the other to image the line that results from the intersection between the beam waist and the object along one axis of the CCD sensor, denoted here the position axis. The remaining axis on the CCD array samples different wavelengths as diffracted by the grating. A highly repeatable tilting mirror (TM) (PI piezoelectric closed loop tilt stage, S-334) is switched between two angular positions in order to illuminate the object from symmetric directions about the observation direction in the plane $y z$. The focal lengths of lenses cL2, L4, cL1 and L3 are $250 \mathrm{~mm}, 150 \mathrm{~mm}, 160 \mathrm{~mm}$ and $100 \mathrm{~mm}$, respectively. In order to validate the inplane depth-resolved displacements measured with this system, a simple shear experiment was performed. In simple shear, for small deformations, the displacement field has a constant in-plane gradient with virtually no out-of-plane component. The sample, S in Fig. 2, consisted of a transparent water based polymer rubber layer with refractive index $n_{2}=1.48$ cured between two glass plates $\mathrm{G}_{1}$ and $\mathrm{G}_{2}$ with refractive index $n_{1}=1.51$. $\mathrm{G}_{2}$ was firmly fixed to a rigid support and $\mathrm{G}_{1}$ was free to move, just held by the bonding force of the polymer layer. In order to introduce simple shear the second glass plate was displaced a known distance by using a mechanism driven by a micro positioning stage.

For oblique illumination in the plane $y z$ at an angle $\theta$ to the $\mathrm{z}$ axis, the phase difference due to object deformation is [8]: 
$\Delta \phi(x, y, z)=\frac{2 \pi}{\lambda}\left[v(x, y, z) n_{0} \sin \theta+w(x, y, z) n_{1}\left(1+\cos \theta_{r}\right)\right]$

where $v(x, y, z)$ and $w(x, y, z)$ are the displacements along the $y$ and $z$ axes and $\theta_{r}$ is the angle of incidence of the refracted beam in the air/glass interface with refractive indexes $n_{0}$ and $n_{1}$, respectively. By choosing equal and opposite illumination angles $\theta$ and $-\theta$, two phase differences can be evaluated, the sum of which gives the out-ofplane component $\Delta \phi_{z}$ and the difference of which gives the in-plane component $\Delta \phi_{y}$. The in-plane and out-of-plane displacements are thus obtained as:

$$
\begin{aligned}
& v(x, y, z)=\frac{\lambda \Delta \phi_{y}(x, y, z)}{4 \pi n_{0} \sin \theta} \\
& w(x, y, z)=\frac{\lambda \Delta \phi_{z}(x, y, z)}{4 \pi n_{1}\left(1+\cos \theta_{r}\right)} .
\end{aligned}
$$

According to Eqs. (2) and (3), with $\theta=5.7^{\circ}$ and $\theta_{r}=3.7^{\circ}$ the resulting out-of-plane and in-plane sensitivities were 0.14 and $4.2 \mu \mathrm{m}$ per fringe, respectively. Refraction at the glass/elastomer interface changes the out of plane sensitivity by only $2 \%$ due to the slight variation in refractive indexes $n_{1}$ and $n_{2}$. With the object in a reference unloaded state, two interferograms were first recorded for each illumination direction. Simple shear was then introduced by moving the non-supported glass plate parallel to itself by a known distance and two more interferograms were recorded, again for the same illumination angles. The closed loop position control of the tilting mirror that switches between one illumination direction and the other, guaranteed a repeatability better than \pm 0.125 mrad. Once recorded, the interferograms were processed as follows. First, the wavelength axis was converted to wave number $k=2 \pi / \lambda$. The interferograms were then Fourier transformed along the $k$-axis to obtain the scattering potential in the illuminated cross section in the object. The phase differences due to depth-dependent 
shear displacements inside the polymer were evaluated for both illumination directions from the Fourier transformed interferograms [9]. One effect of the oblique illumination is a tilt of the reconstructed cross section of the object in the $y z$ plane, reflecting the tilt between the zero delay reference plane and the oblique wavefronts illuminating the object. This tilt is removed by re-referencing the scattering potential corresponding to each illumination direction [8]. The out-of-plane and in-plane phase components are then obtained by adding and subtracting, respectively, the reregistered phase differences for each illumination direction. Figure 3(a) shows the inplane wrapped phase map for the simple shear test. As expected, a linear phase profile is observed in the region between glass plates $G_{1}$ and $G_{2}$, with virtually no phase change for $\mathrm{G}_{2}$, indicating that it remained static. Figure 3(b) shows the wrapped phase corresponding to the out-of-plane sensitivity. The thickness of the polymer layer was $400 \mu \mathrm{m}$, thin compared with its $\sim 20 \mathrm{~mm}$ diameter in the $x y$ plane. This reduced to a very small level the out-of-plane displacements due to bending moments whilst moving $\mathrm{G}_{1}$ along the $y$-axis. Figure 4 shows the corresponding displacement profiles obtained by averaging the displacement fields obtained from Figs. 3(a) and 3(b) and the corresponding sensitivities for each phase component along the position axis between $y=1.7$ and $3.7 \mathrm{~mm}$ for optical depth values between $z_{o}=2.3$ and $2.9 \mathrm{~mm}$. The range of $y$ values chosen corresponds to the region where the illumination beams had intensity levels closer to the maximum, thus resulting in a higher phase SNR. The displacement introduced with the micrometer screw driven stage, $1.6 \pm 0.1 \mu \mathrm{m}$, is indicated in Fig. 4. Noting that the two lines running from ( $\left.v=0 \mu \mathrm{m}, z_{o}=2.9 \mathrm{~mm}\right)$ to $\left(v=1.6 \pm 0.1 \mu \mathrm{m}, z_{o}=2.3 \mathrm{~mm}\right)$ represent the maximum and minimum in-plane displacement profiles expected within the polymer layer due to simple shear, it is observed that the measured displacements are well within the expected values. For the 
in-plane displacements an RMS error of $\sim 6 \%$ was found between the optical measurements and the expected average displacement profile, probably due to speckle noise and a slight non-linearity in the displacement field gradient.

In conclusion, the ability of phase contrast spectral domain OCT to measure depthresolved displacements within a weakly scattering material was demonstrated using a system based on oblique illumination. Both in-plane and out-of-plane displacement components are obtained, as opposed to conventional OCT systems in which normal illumination can only lead to out-of-plane sensitivity.

\section{Acknowledgements}

The authors are grateful to the Leverhulme Trust and Dr. Fernando Mendoza for supporting this research and to Dr. Gustavo Galizzi for his technical assistance. 


\section{References}

[1] 1. P. Rastogi, Optical Measurement Techniques and Applications, (The Artech house publishers, 1997).

[2] A. F. Fercher, W. Drexler, C. K. Hitzenberger and T. Lasser, “Optical coherence tomography -principles and applications,” Rep. Prog. Phys. (66), 239 (2003).

[3] G. Gülker, K. D. Hinsch and A. Kraft, "Low coherence ESPI in the investigation of ancient terracotta warriors,” in Speckle Metrology 2003, K. Gastinger, O. J. Løckberg and S. Winther, eds., Proc. SPIE 4933, 53 (2003).

[4] K. Gastinger, S. Winther and K. D. Hinsch, "Low-coherence speckle interferometer (LCSI) for characterization of adhesion in adhesive-bonded joints,” in Speckle Metrology 2003, K. Gastinger, O. J. Løkberg, and S. Winther, eds., Proc. SPIE 4933, 59 (2003).

[5] P. D. Ruiz, Y. Zhou, J. M. Huntley and R. D. Wildman, “Depth-resolved whole field displacement measurement using wavelength scanning interferometry,” J. Opt. A: Pure Appl. Opt. 6, 679 (2004).

[6] P. D. Ruiz, J. M. Huntley and R. D. Wildman, “Depth-resolved whole-field displacement measurements by wavelength-scanning electronic speckle pattern interferometry,” Appl. Opt. 44, 3945 (2005). 
[7] Manuel De la Torre Ibarra, Pablo D. Ruiz and Jonathan M. Huntley, “Double-shot depth-resolved displacement field measurement using phase contrast spectral optical coherence tomography”, Optics Express (14) 9643 (2006)

[8] Pablo D. Ruiz, Jonathan M. Huntley and Alejandro Maranon, “Tilt scanning interferometry: a novel technique for mapping structure and three-dimensional displacement fields within optically scattering media”, Proc. R. Soc. A (462), 2481 (2006)

[9] J. M. Huntley, “Automated Analysis of Speckle Interferograms,” in Digital Speckle Pattern Interferometry and Related Techniques, P. K. Rastogi ed., (Chichester, West Sussex, England, John Wiley \& Sons., 2001) pp. 59-139. 


\section{Figure captions}

Figure 1. a) Top view of a phase-contrast spectral OCT system for measuring displacements with dual sensitivity showing: fixed collimators L1, L2, cylindrical lenses cL1, cL2, spherical lenses L3, L4, neutral density filter NF, tilting mirror TM, beam splitter BS, grating $\mathrm{G}$ and sample S. b) Unfolded side view of the illumination beam.

Figure 2. Oblique illumination of polymer layer $\mathrm{S}$ constrained between two glass plates $\mathrm{G}_{1}$ and $\mathrm{G}_{2}$

Figure 3. In-plane (a) and out-of-plane (b) wrapped phase (black and white represent $-\pi$ and $\pi$, respectively) due to simple shear of a polymer layer sandwiched between two glass plates. The regions between 0 and $1 \mathrm{~mm}$ and between $\sim 2.2$ and 3mm correspond to the cross correlation terms and to the polymer layer, respectively. .

Figure 4. In-plane and out-of-plane displacement profiles through the thickness of a polymer layer under pure shear. 
(a)

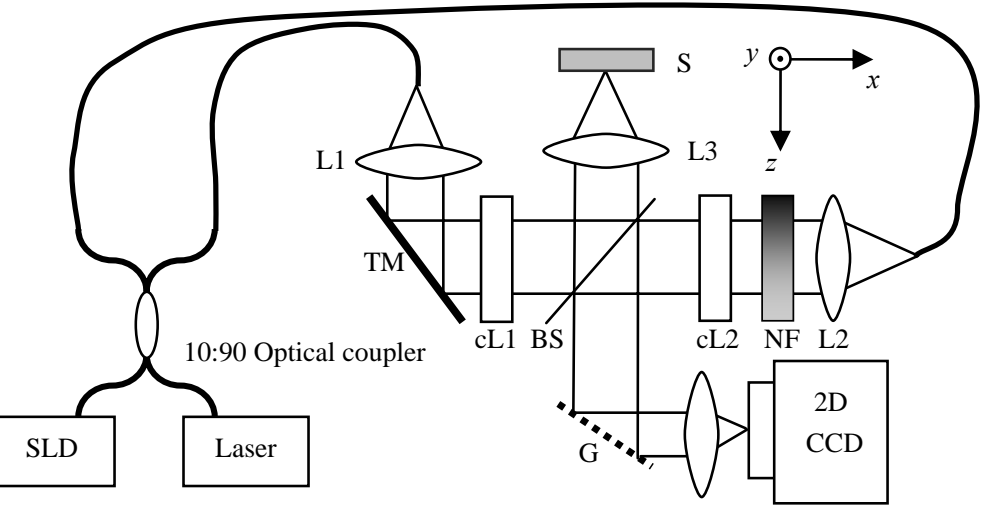

(b)

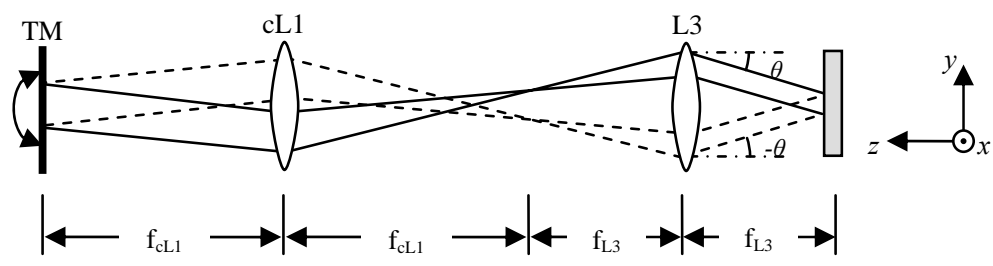

Figure 1 


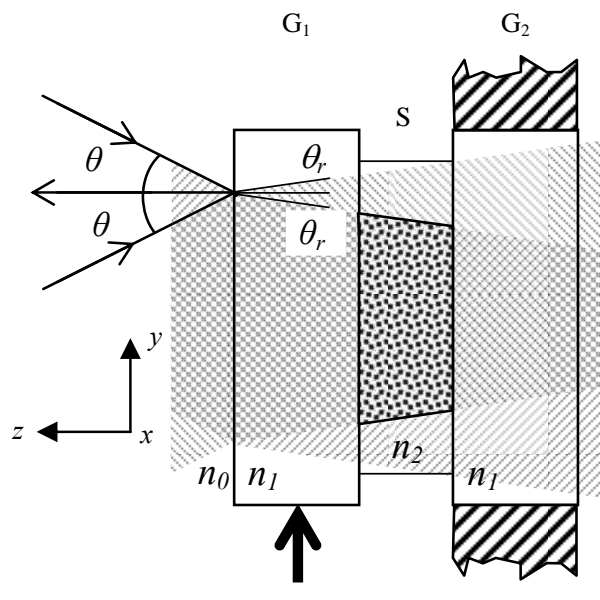

Figure 2 


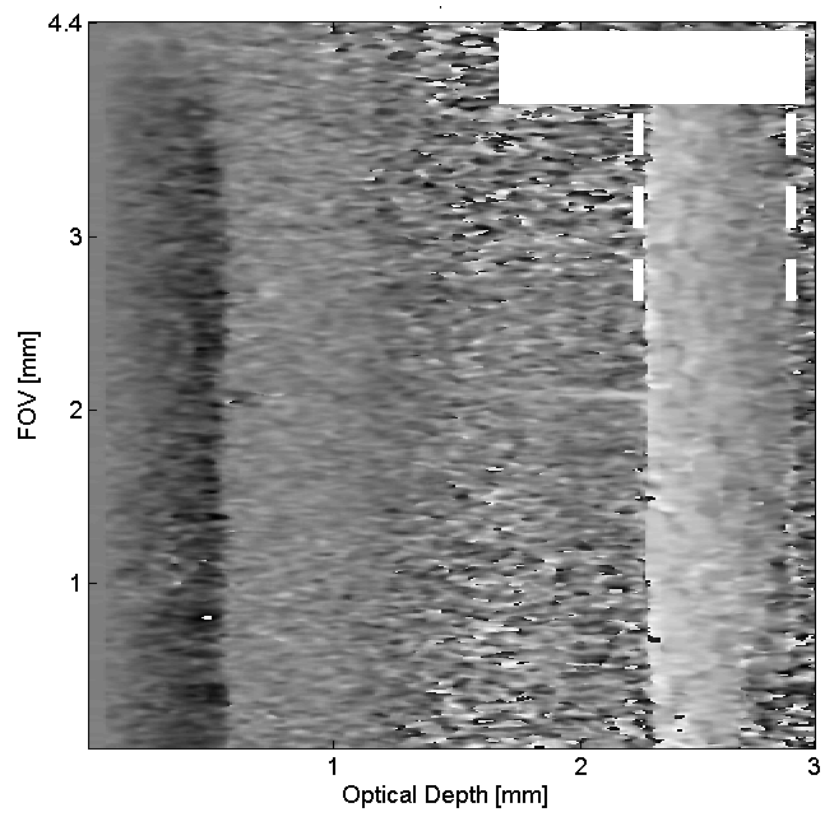

(a)

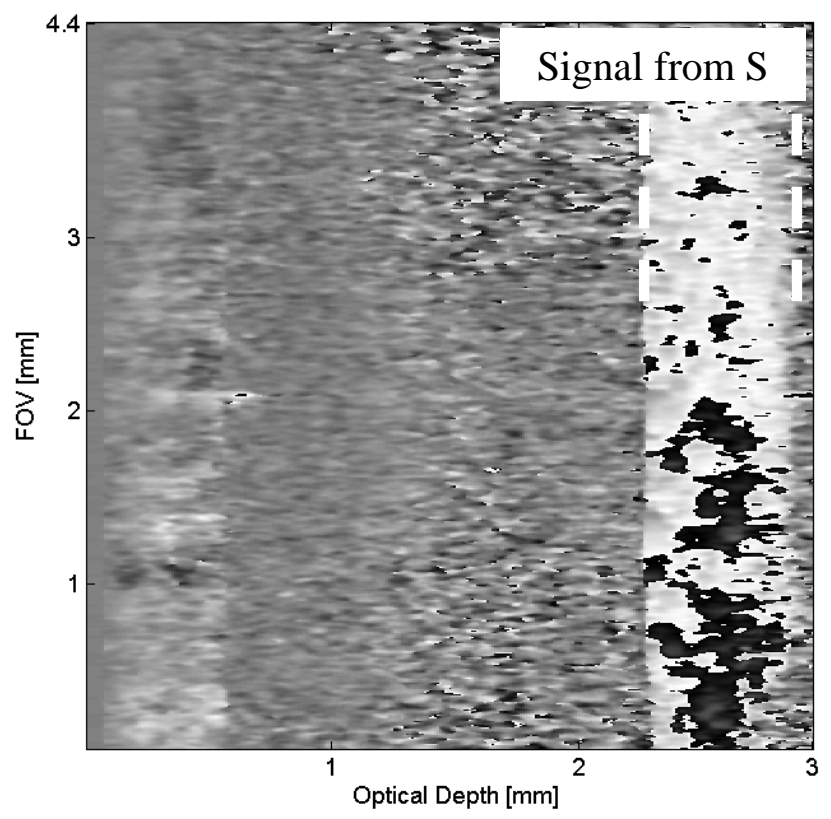

(b)

Figure 3 


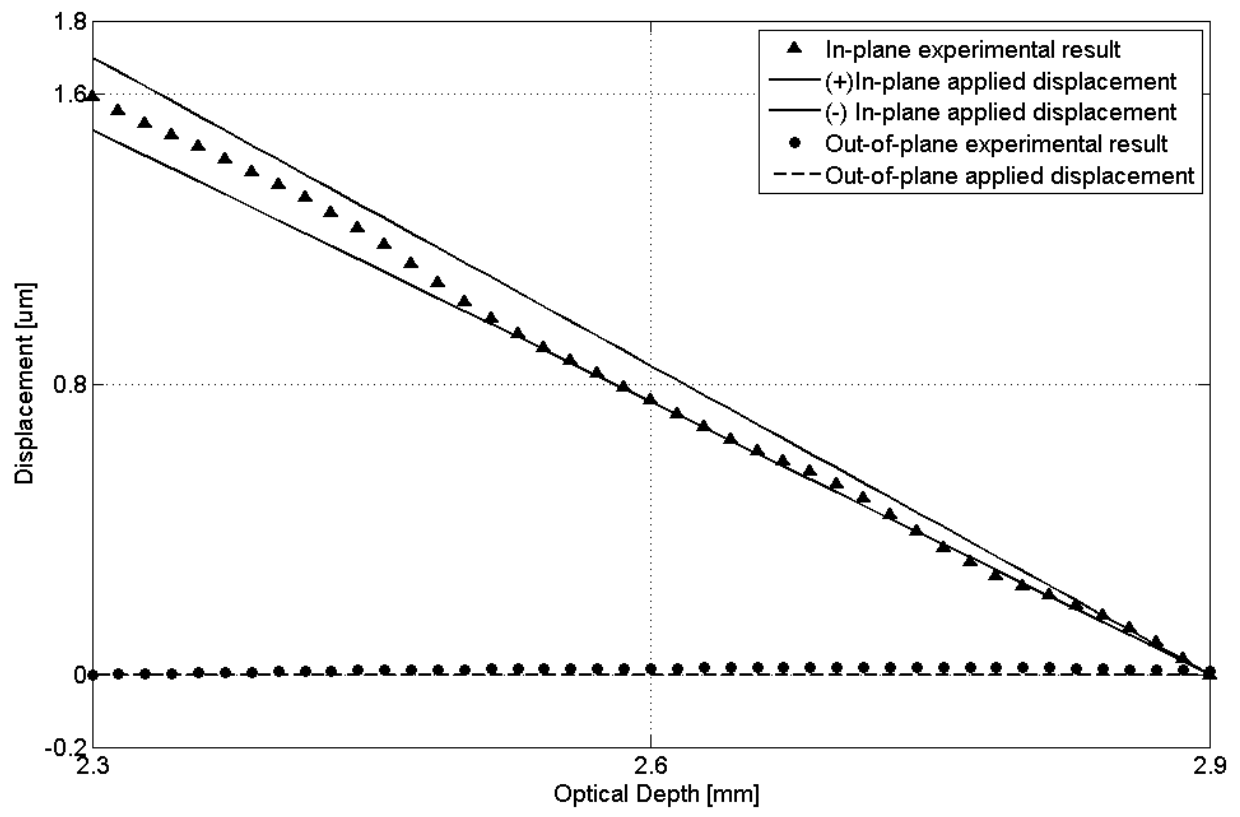

Figure 4 